\title{
O Papel da Capacitação em Saúde nos Resultados da Baciloscopia para Hanseníase
}

\author{
The Role of Health Training in the Results of Skin Smears for Leprosy
}

\author{
Susilene Maria Tonelli Nardi ${ }^{1}$ \\ Valter Batista Duo Filho \\ Naiara Cristina Ule Belotti ${ }^{2}$ \\ Flávia dos Santos Patine ${ }^{3}$ \\ Tânia Maria Araujo ${ }^{4}$ \\ Vania Del'Arco Paschoal ${ }^{5}$ \\ Fernanda Modesto Tolentino Binhardi ${ }^{6}$ \\ Heloisa da Silveira Paro Pedro ${ }^{7}$
}

\section{RESUMO}

Objetivo: Comparar o resultado e as inconsistências das baciloscopias nos períodos pré e pós-capacitações sobre baciloscopia. Metodologia: As informações sobre as baciloscopias foram obtidas no Instituto Adolfo Lutz de São José do Rio Preto-SP (IAL-SJRP), no período de 2010 a 2014 , quando não ocorreu capacitação e de 2015 a 2018, quando foram realizadas cinco capacitações e foram comparados os resultados dos períodos. Resultados: Capacitaram-se 205 profissionais: enfermeiros $(64 \%)$ e técnicos/auxiliares de enfermagem $(18 \%)$, sendo os profissionais da atenção básica os que mais participam $(p<0,05)$. Foram enviadas ao IAL-SJRP $1375(52,9 \%)$ lâminas antes e 1224 (47,1\%) após as capacitações. A positividade dos exames subiu de $7 \%(n=97)$ para $16,2 \%(n=196)(p<0,05)$. A média do índice baciloscópico antes capacitação foi de 1,67 (DP 0,98) e após 1,92 (DP 1,01) $(p<0,05)$. O material escasso $(31,5 \%)$ e a coleta inadequada $(47,3 \%)$ foram as inconsistências mais frequentes, as quais diminuíram $26,6 \%$ pós-treinamento. Conclusões: A capacitação mostrou-se eficaz, aumentando a positividade das baciloscopias, tendo sido padronizados os procedimentos e, consequentemente, diminuído as inconsistências.

\section{DESCRITORES}

Hanseníase. Mycobacterium Leprae. Laboratórios. Capacitação em Serviço.

\begin{abstract}
Objective: To compare the results and failures of skin smears for leprosy before and after training for skin smear. Methodology: Information on skin smears was obtained at the Instituto Adolfo Lutz of São José do Rio Preto-SP city from 2010 to 2014, when there was no training and from 2015 to 2018 , when five training sessions were held. Results: 205 professionals were trained. Primary health care nurses (64\%) and nursing technicians (18\%) were the ones that mostly participated ( $p$-value $<0.05) .1,375$ slides were sent (52.9\%) before training and 1,224 (47.1\%) after it. The tests' positivity raised from $7 \%(n=97)$ to $16.2 \%(n=196)$ (p-value $<0.05$ ). The Bacterial Index before training was 1.67 (SD $0.98)$ and after $1.92(\mathrm{SD} 1.01)$ ( $p$-value $<0.05)$. Scarce material $(31.5 \%)$ and inadequate collection $(47.3 \%)$ were the most frequent failures in tests, that decreased $26.6 \%$ after training Conclusions: The training proved to be effective, increased the positivity of skin smears, standardized the procedures and decreased failures.
\end{abstract}

\section{DESCRIPTORS}

Leprosy. Mycobacterium Leprae. Laboratories. In-service Training.

\footnotetext{
${ }^{1}$ Terapeuta Ocupacional, Doutora, Instituto Adolfo Lutz, São José do Rio Preto, São Paulo, Brasil.

2 Biomédico(a), Especialista em Microbiologia, Instituto Adolfo Lutz, São José do Rio Preto, São Paulo, Brasil.

${ }^{3}$ Mestre, Grupo de Vigilância Epidemiológica 29- GVE-29, São José do Rio Preto, São Paulo, Brasil.

${ }^{4}$ Enfermeira, Especialista em Saúde Pública, Prefeitura Municipal de Ubarana, São Paulo, Brasil.

${ }^{5}$ Enfermeira, Doutora, Faculdade de Medicina de São José do Rio Preto - FAMERP, São José do Rio Preto, São Paulo, Brasil.

${ }^{6}$ Biomédica, Doutora, Instituto Adolfo Lutz, São José do Rio Preto, São Paulo, Brasil.

${ }^{7}$ Bióloga, Doutora, Instituto Adolfo Lutz, São José do Rio Preto, São Paulo, Brasil.
} 
A hanseníase é causada pelo Mycobacterium leprae, um bacilo álcool-ácido resistente (BAAR), que tem predileção pela pele e pelos nervos periféricos. A demora no diagnóstico e tratamento dos indivíduos pode acarretar incapacidades e colocar em risco de contaminação aqueles que têm convívio diário com o indivíduo que não iniciou o tratamento ${ }^{1}$.

A taxa de detecção de 12,2/100 mil hab. no Brasil coloca-o no ranking de $2^{\circ}$ país mais endêmico do mundo ${ }^{2}$.

O diagnóstico da hanseníase é clínico-epidemiológico, não existindo exame laboratorial padrão-ouro ${ }^{1}$. A baciloscopia do raspado intradérmico é um procedimento que exige profissionais bem treinados para sua execução e, se o exame for positivo, define o diagnóstico, a classificação da forma clínica e a condução de terapêutica ${ }^{3,4}$.

A baciloscopia é um exame de baixa complexidade que consiste na coleta de raspado intradérmico em sítios estabelecidos pelo Ministério da Saúde (lóbulos auriculares, cotovelos e lesões suspeitas), mas que necessita de estrutura e capacitação para sua execução devido às singularidades dessa prática, uma vez que ainda existe dificuldade no processo de coleta, fixação, transporte, coloração e mesmo na leitura de lâminas de baciloscopia ${ }^{1}$.

No Brasil, o Programa Nacional de Controle da Hanseníase (PNCH) estabelece normativas e impõe ações de saúde, como os treinamentos ${ }^{5,6}$ para tentar reverter a atual situação endêmica da doença, porém, com resultados ainda insatisfatórios ${ }^{7}$.

Devido à complexidade da doença, a falta da avaliação da efetividade do treinamento, a pouca disponibilidade de tempo, a alta rotatividade dos profissionais e a escassez de informações fazem com que os profissionais sintam-se despreparados para seguir o protocolo de diagnóstico da hanseníase ${ }^{8,10}$.

Visando à saúde pública, estratégias consistentes devem ser elaboradas pensando não só na capacitação profissional, mas na estruturação das ações de controle da hanseníase, para que sejam duradouras e apresentem resultados satisfatórios diante dessa endemia ${ }^{8,10,11}$.

Sendo assim, o objetivo desse estudo foi comparar o resultado e inconsistências das baciloscopias nos períodos anterior e posterior às capacitações, realizadas para profissionais de saúde de municípios pertencentes ao Grupo de Vigilância Epidemiológica - 29, de São José do Rio Preto - SP (GVE-29).

\section{METODOLOGIA}

As informações sobre as baciloscopias foram obtidas no livro de registros de exames laboratoriais do Instituto Adolfo Lutz de São José do Rio Preto - SP (IAL-SJRP), referência laboratorial para 48 dos 67 municípios do GVE-29.

Os dados relativos às capacitações foram coletados nas listas de presença com informações sobre os municípios participantes, datas das capacitações, $\mathrm{n}^{\circ}$ de profissionais que participaram e respectivas formações profissionais. Cada capacitação abordou os temas coleta de raspado intradérmico, padronização do formulário de coleta/ identificação do paciente e a importância do transporte, do armazenamento, da 
coloração pelo método de Ziehl-Neelsen à frio, leitura dos esfregaços e interpretação dos resultados laboratoriais de forma teóricoprática, ministrados por uma enfermeira e por bióloga e biomédica.

As variáveis utilizadas para as baciloscopias foram coletadas nos livros de registros de exames do laboratório em dois períodos distintos: de 2010 a 2014, quando não tinha sido realizada nenhuma capacitação e, de 2015 a 2018, período que contemplou cinco capacitações. Foram compilados o número de baciloscopias realizadas, se a coleta correspondia ao diagnóstico ou controle de tratamento do paciente, o número dos sítios coletados, os resultados das baciloscopias e o índice baciloscópico, além das seguintes inconsistências das lâminas que estavam descritas no livro de registro do laboratório: material escasso, coleta inadequada (esfregaço fora do padrão recomendado ou sem os 4 esfregaços na mesma lâmina), um ou mais esfregaço invertido na lâmina, material sanguinolento e identificação do paciente incorreta. Todas as informações foram registradas no banco de dados. Os resultados foram comparados considerando os profissionais que passaram ou não por capacitação. Os dados foram inseridos em planilhas e analisados no software Epilnfo ${ }^{\mathrm{TM}} 7^{12}$, com descrição das frequências, médias e desvios-padrão e, para associação das variáveis, utilizou-se os testes de Qui-quadrado, Kruskal-Wallis e exato de Fisher, quando apropriados.

Este estudo foi aprovado pelo Comitê de Ética e Pesquisa do Instituto Adolfo Lutz com o Parecer $n^{\circ}$ 2.101.044.

\section{RESULTADOS}

Dos 48 municípios do Grupo de Vigilância Epidemiológica 29 - GVE-29, dos quais o IAL-SJRP é referência laboratorial, $38(79,2 \%)$ enviaram profissionais para serem capacitados nos cursos de baciloscopia oferecidos no período de 2015 a 2018. Outros 10 municípios, dos quais o IAL-SJRP não é referência, enviaram 21 profissionais para serem capacitados neste período (Figura 1).

No período de 2015 a 2018 foram capacitados 205 profissionais, porém, 184 deles pertenciam aos municípios que o IALSJRP é referência laboratorial. A formação destes profissionais e o tipo de serviço a que eles pertenciam estão representados na Tabela 1.

Os enfermeiros e os técnicos/auxiliares de enfermagem que atuam na atenção básica são os que mais procuram pelos cursos de baciloscopia em hanseníase quando comparado aos demais profissionais $(p<0,05)$

Nos períodos estudados foram enviadas ao IAL-SJRP 2599 baciloscopias, sendo 1375 (52,9\%) antes das capacitações e $1224(47,1 \%)$ após.

De 2010 a 2014, foram positivos 97 exames (7\%), de 2015 a 2018, 196 (16,2\%), evidenciando um aumento da positividade após as capacitações realizadas $(p=0,000)$ (Figura 2).

Dos 196 exames positivos no período em que ocorreu capacitação, 166 (84,7\%) foram coletados por profissionais treinados.

Antes dos treinamentos, a média de esfregaços por paciente estava abaixo do indicado pelo protocolo de coleta (média de $3,4)$, com apenas 415 baciloscopias (30\%) 
Figura 1. Mapa dos municípios do Grupo de Vigilância Epidemiológica XXIX - GVE-29 que enviaram profissionais para serem capacitados para coleta de Baciloscopia em hanseníase

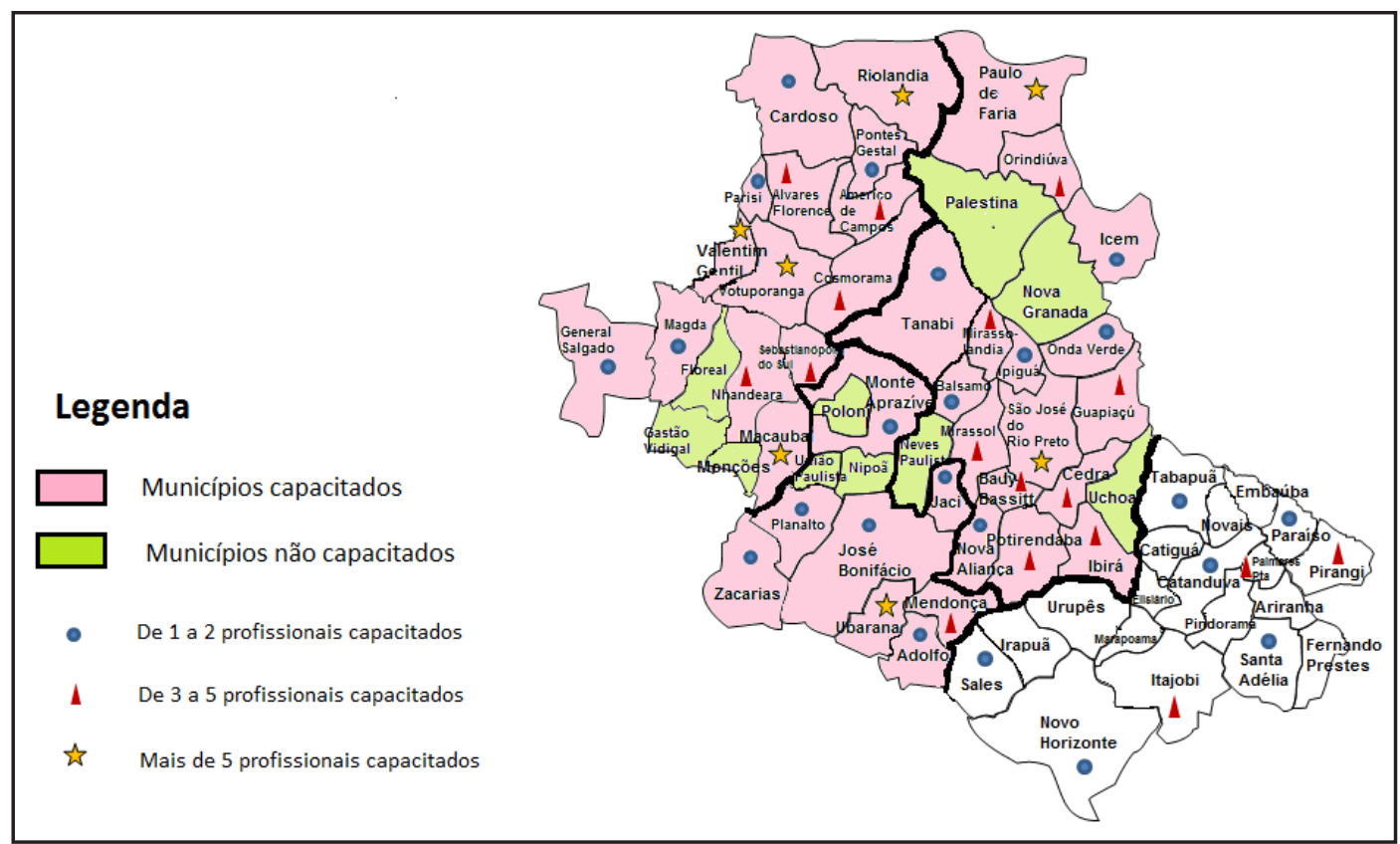

Tabela 1. Distribuição dos profissionais capacitados e respectivos serviços de atuação

\begin{tabular}{|c|c|c|c|c|c|c|c|c|c|c|}
\hline \multirow[t]{2}{*}{ Formação } & \multicolumn{2}{|c|}{$\begin{array}{l}\text { Atenção } \\
\text { Básica }\end{array}$} & \multicolumn{2}{|c|}{$\begin{array}{l}\text { Atenção } \\
\text { Secundária }\end{array}$} & \multicolumn{2}{|c|}{$\begin{array}{l}\text { Atenção } \\
\text { Terciária }\end{array}$} & \multicolumn{2}{|c|}{$\begin{array}{c}\text { Vigilância } \\
\text { Epidemiológica }\end{array}$} & \multicolumn{2}{|c|}{ Total } \\
\hline & $\mathrm{n}$ & $\%$ & $\mathrm{n}$ & $\%$ & $\mathrm{n}$ & $\%$ & $\mathrm{n}$ & $\%$ & $\mathrm{n}$ & $\%$ \\
\hline Enfermeiros & 97 & 52,7 & 03 & 1,6 & 05 & 2,7 & 10 & 5,4 & 115 & 62,4 \\
\hline $\begin{array}{l}\text { Técnicos e ou } \\
\text { auxiliares de } \\
\text { enfermagem }\end{array}$ & 26 & 14,2 & 02 & 1,1 & 02 & 1,1 & 04 & 2,2 & 34 & 18,6 \\
\hline Biomédicos & 03 & 1,6 & 13 & 7,1 & - & - & - & - & 16 & 8,7 \\
\hline Biólogo & - & - & 05 & 2,7 & - & - & - & - & 05 & 2,7 \\
\hline Médicos & 03 & 1,6 & 02 & 1,1 & - & - & - & - & 05 & 2,7 \\
\hline $\begin{array}{l}\text { Auxiliares de } \\
\text { laboratório }\end{array}$ & 01 & 0,5 & 01 & 0,5 & 01 & 0,5 & - & - & 03 & 1,6 \\
\hline $\begin{array}{l}\text { Outros profissionais } \\
\text { de saúde* }\end{array}$ & 04 & 2,2 & 02 & 1,1 & - & - & - & - & 06 & 3,3 \\
\hline Total & 134 & 72,8 & 28 & 15,2 & 08 & 4,3 & 14 & 7,6 & 184 & 100,0 \\
\hline
\end{tabular}

*Outros profissionais de saúde: 02 Fisioterapeutas; 02 Químicas; 01 Farmacêutico; 01 Bioquímica; 01 Auxiliar Administrativo. 
no padrão de quatro esfregaços; depois das capacitações, a média seguiu o protocolo (média de 4) com 1094 baciloscopias (89,3\%) atendendo ao padrão, sendo 865 delas $(79,1 \%)$ oriundas de profissionais capacitados.

Após as capacitações, a média do índice baciloscópico (IB) foi de 1,92 $\pm 1,01$

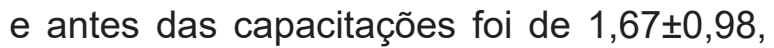
sendo este aumento significante $(p<0,05)$.

Das lâminas recebidas no período, $72,2 \%$ foram para diagnóstico, 25,5\% para controle de tratamento e 2,3\% sem informação.
Ao analisarmos as baciloscopias positivas no diagnóstico e no controle de tratamento, a positividade foi significantemente maior no controle de tratamento quando coletada pelos profissionais capacitados $(p<0,05)$ (Tabela 2).

Em relação às inconsistências nas lâminas coletadas, no período sem capacitação, as mais frequentes foram material escasso $(31,5 \%)$ e coleta inadequada $(47,3 \%)$. Após as capacitações, as inconsistências ainda estavam presentes, porém, ocorreu uma queda de $26,6 \%$ das lâminas recebidas com a coleta inadequada.

\section{Figura 2. Positividade das baciloscopias de acordo com o ano de coleta}

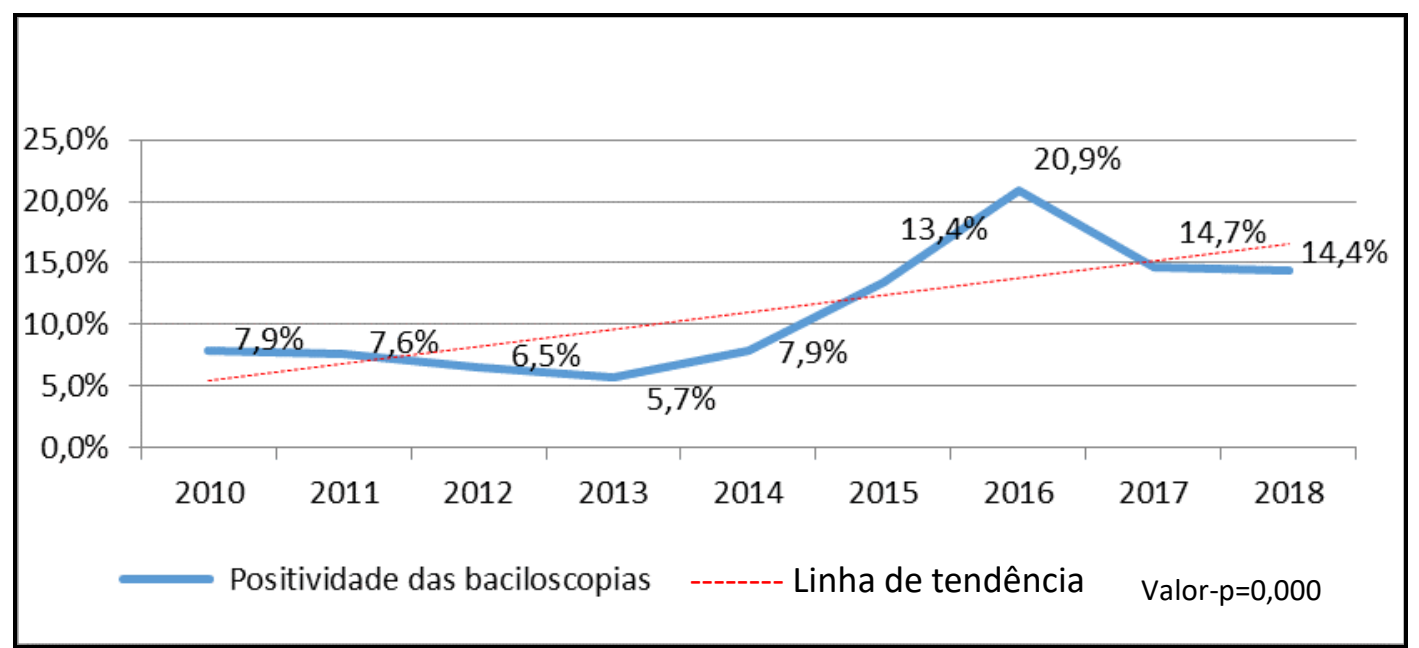

Tabela 2. Baciloscopias positivas de acordo com o momento da coleta e a capacitação profissional

\begin{tabular}{|c|c|c|c|c|c|c|}
\hline \multirow{3}{*}{$\begin{array}{l}\text { Profissional } \\
\text { Capacitado }\end{array}$} & \multicolumn{6}{|c|}{ Momento da coleta e positividade das baciloscopias } \\
\hline & \multicolumn{2}{|c|}{ Diagnóstico } & \multicolumn{2}{|c|}{ Controle de tratamento } & \multirow[b]{2}{*}{ Total } & \multirow[b]{2}{*}{ Valor-p* } \\
\hline & $\mathrm{n}$ & $\%$ & $\mathrm{n}$ & $\%$ & & \\
\hline Não & 82 & 66,7 & 41 & 33,3 & 123 & \multirow{2}{*}{$<0,05$} \\
\hline Sim & 50 & 30,3 & 115 & 69,7 & 165 & \\
\hline
\end{tabular}

\footnotetext{
${ }^{*}$ Chi-square - corrected (Yates)
} 
Realizaram-se anotações no livro de registros do laboratório de referência, identificando inconsistências em algumas lâminas enviadas pelos profissionais ao longo do período, como má coleta, lâminas sanguinolentas, esfregaço invertido, sem especificação do sitio de coleta. Do total de 66 lâminas com inconsistências, 41 (68,3\%) vieram de profissionais que não tinham sido capacitados.

\section{DISCUSSÃO}

A endemia da hanseníase apresentase de forma heterogênea no país e, embora, $51,0 \%$ dos municípios do Estado de São Paulo apresentem detecção igual a zero, $20,0 \%$ deles ainda tem detecção maior de $10,0 / 100.000$ habitantes $^{13}$, fato esse que preserva a doença circulante ${ }^{14}$. Importante ressaltar que a região desse estudo fica na fronteira com o Estado de Mato Grosso do Sul, que apresenta municípios com detecção alta de casos e, embora, nossa região tenha atingido os parâmetros de eliminação da doença (menos de 1 caso a cada 10.000 habitantes), no entanto, se não existir ações de vigilância epidemiológica, poderá ocorrer uma reversão desfavorável destes indicadores. Alguns municípios da região desse estudo, curiosamente, na região de fronteira com o Estado do Mato Grosso do Sul, ainda apresentam índices desfavoráveis da doença. De acordo com a PES (2016-2019), $100 \%$ dos municípios do Estado devem ser acompanhados para o alcance ou sustentação da meta de eliminação da hanseníase ${ }^{13}$.
A capacitação de profissionais em suas respectivas áreas de atuação, com ênfase nos problemas que acontecem no dia a dia, é importante no sentido de melhor atender as necessidades dos usuários e do serviço de saúde, aumentando a qualificação do SUS ${ }^{15,16}$, além de contribuir para mudanças na prática profissional ${ }^{17,20}$, com resultados sempre positivos. Para alcançar as metas de eliminação da hanseníase, deverá de se investir em estruturação das ações de controle e capacitação de profissionais de saúde, desde a sua graduação ${ }^{21}$. Importante ressaltar que o profissional presente na capacitação deve estar inserido e envolvido na atividade em questão, para que haja transformação de suas ações e eficácia do serviço ${ }^{22}$.

O primeiro curso de capacitação da coleta de material para a baciloscopia de hanseníase, com parceria do IAL com o Grupo de Vigilância Epidemiológica-29, foi organizado buscando melhorar a qualidade dos exames coletados e recebidos no laboratório, devido aos inúmeros erros e falhas relacionados à coleta de raspado intradérmico, para obter melhor qualidade em todas as etapas do exame até o resultado final. Foi planejado para ser participativo e prático, buscando atender as necessidades das instituições e a valorização dos profissionais envolvidos.

O fato dos enfermeiros serem os principais profissionais que participam dos cursos de capacitação, se explica pelos mesmos serem os primeiros contatos nas unidades de saúde com os pacientes ${ }^{15} \mathrm{e}$, também, os principais profissionais que 
realizam ações para o diagnóstico e o controle do tratamento da hanseníase ${ }^{23,24}$. Outros profissionais são advindos de órgãos públicos, na busca de capacitação e/ou aperfeiçoamento na área de hanseníase.

A melhor qualidade na coleta de raspado intradérmico proporciona o aumento de positividade de baciloscopias, ao melhorar a qualidade do resultado na visualização de bacilos e outras estruturas, o que fica evidenciado pelo aumento de resultados positivos coletados por profissionais capacitados, após o período de capacitações e, também, ao analisar o aumento do índice baciloscópico no período pós-capacitações. De fato, quando submetidos à coloração, os bacilos apresentam-se corados, geralmente de modo irregular, podendo apresentar-se com diversas formas ${ }^{25}$. A visualização dos bacilos fragmentados e, principalmente dos granulosos, na microscopia é muito mais difícil quando o material é escasso na lâmina.

As capacitações para os profissionais de saúde protocolam rotinas e processos das mais variadas áreas quando pensamos em um ambiente de saúde pública, o que acarreta resultados melhores e mais confiáveis ${ }^{15}$. Além do aumento da positividade, os resultados desse estudo apontam que no período após as capacitações evidenciou-se o aumento da padronização da coleta de quatro esfregaços, normatizadas pelo manual de baciloscopia em hanseníase ${ }^{25}$ e a diminuição das inconsistências encontradas nas lâminas recebidas, mostrando a resposta positiva imediata dos procedimentos realizados pelos profissionais quando existiu a capacitação.
Ressaltamos que um fator comumente encontrado nas unidades públicas é a rotatividade dos profissionais ${ }^{10,26}$, que tem se tornado uma realidade constante devido às necessidades de cumprimento de outros programas/agendas que surgem dentro das unidades de saúde, ou ainda, pela escassez de recursos humanos que acabam por ocasionar uma lacuna no serviço e, esse fato, não pode ser negligenciado. Além disso, existe também a entrada de novos profissionais para atuação nos serviços do SUS ${ }^{16}$. Mais uma vez, a necessidade de capacitações frequentes se faz imperativa.

Organizar a rede com capacitações numa região que tem uma grande quantidade de municípios e profissionais com formações variadas, conhecimentos diferentes e instituições distintas, com competências diferenciadas, não é uma tarefa fácil, mas fundamental para colaborar com o diagnóstico/ controle de tratamento da hanseníase. Avaliar os resultados obtidos na prática laboratorial após os treinamentos profissionais é essencial para verificar o impacto real do trabalho realizado em conjunto ${ }^{16,18}$.

Embora a elaboração de educação continuada com capacitações não seja uma idéia inovadora, uma vez que tem ocorrido em todas as esferas do SUS ${ }^{27,28}$, a análise dos resultados obtidos na prática, com vistas a melhorar a qualidade da baciloscopia no laboratório é inédita.

Devido à escassez de estudos com esse escopo, a principal limitação encontrada foi que a busca na literatura resultou em poucos artigos com resultados das baciloscopias 
após as capacitações em saúde na área de hanseníase, onde os resultados pudessem ser comparados aos encontrados.

Ações para um trabalho integrado entre o laboratório, a vigilância epidemiológica e as unidades de saúde poderão contribuir para que a padronização dos processos de coleta, podendo aumentar a qualidade da baciloscopia, do exame laboratorial rápido, de baixo custo e pouco invasivo disponível atualmente na rede publica, para colaborar com o diagnóstico da hanseníase ${ }^{25}$.

\section{CONCLUSÃO}

Foram capacitados 205 profissionais e, dos 48 municípios dos quais o IAL é referência para a baciloscopia da hanseníase, 38 (79,2\%) enviaram profissionais para serem capacitados nos cursos de baciloscopia.

Profissionais da área de enfermagem, enfermeiros, técnicos e auxiliares de enfermagem que atuam na atenção básica foram os que mais procuram pelos cursos de baciloscopia em hanseníase quando comparado aos demais profissionais.

Após as capacitações realizadas, ocorreu um aumento significante da positividade da baciloscopia, sendo que $84,7 \%$ das lâminas foram coletados por profissionais treinados. Foi significante, também, o aumento da média do índice baciloscópico (IB) após as capacitações.

A positividade foi significantemente maior nas lâminas para o controle de tratamento em relação aquelas coletadas para o diagnóstico da doença, quando coletada pelos profissionais capacitados.

Embora as inconsistências nas lâminas coletadas (material escasso e coleta inadequada) ainda estejam presentes após as capacitações, existiu uma queda de $26,6 \%$ das lâminas recebidas com a coleta inadequada.

A capacitação mostrou-se eficaz, provando um aumento da positividade das baciloscopias, padronização dos procedimentos, diminuição das inconsistências, evitando-se assim, posterior recoleta e/ou descarte de lâminas.

\section{REFERÊNCIAS}

1. Ministério da Saúde (BR). Secretaria de Vigilância em Saúde. Departamento de Vigilância e Doenças Transmissíveis. Guia prático sobre a hanseníase. Brasília: Ministério da Saúde; 2017.

2. Ministério da Saúde (BR). Secretaria de Vigilância em Saúde. Boletim Epidemiológico. Caracterização da situação epidemiológica da hanseníase e diferenças por sexo, Brasil, 2012-2016. Brasília: Ministério da Saúde; 2018. 
3. Nascimento MS, Rodrigues ZL. O Lacen e a importância dos dados laboratoriais de baciloscopias para confirmação do diagnóstico, classificação de casos, acompanhamento e alta do paciente com hanseníase. Revista Intersaberes. 2010; 10(5): 285-312.

4. Crippa ILF, Schettini AP, Pennini SN, Schettini MC, Rebello PFB. Correlação clínico-laboratorial baseada em dados secundários dos casos de hanseníase atendidos no período de 01/2000 a 03/2001 na Fundação Alfredo da Matta, Manaus-AM, Brasil. An Bras Dermato. 2004; 79(5):547-554.

5. Leroy FS, Coelho ACO, Niitsuma ENA, Gomes FBF, Lanza FM, Ribeiro GC, et al. Educação permanente em saúde: a experiência do uso da educação a distância na capacitação em ações de controle da hanseníase. Em Rede Rev Ed Distância. 2017; 4(1):235-50.

6. Leal DR, Cazarin G, Bezerra LCA, Albuquerque AC, Felisberto E. Programa de Controle da Hanseníase: uma avaliação da implantação no nível distrital. Saúde Debate. 2017.

7. Costa VHMV, Cavalcanti LA, Faria-Junior JAD, Kitaoka EG, Mascarenhas GS, Mascarenhas NB et al. Programa nacional de eliminação da hanseníase: um estudo sobre a avaliabilidade do programa e das suas ações em âmbito estadual e municipal. Rev Baiana Saúde Pública. 2010; 34(3):450-467.

8. Souza ALA, Feliciano KVO, Mendes MFM. A visão de profissionais da Estratégia Saúde da Família sobre os efeitos do treinamento de hanseníase. Rev Esc Enfermagem. 2015; 49(4):610-618.

9. Lanza FM, Lana FCF. Descentralização das ações de controle da hanseníase na microrregião de Almenara, Minas Gerais. Rev. Latino-Am. Enfermagem. 2011; 19(1):08.

10. Beluci ML, Borgato MHB, Galan NGA. Avaliação de cursos multiprofissionais em hanseníase. Hansen Int. [Internet]. 2012; 37(2): 47-53.

11. Gonçalves A. Realidades do controle da hanseníase: atualizando cenários. Rev Bras Epidemiol. 2013; 16(3): 611-21.

12. EPI Info ${ }^{\mathrm{TM}} 7$ [internet]. Version 7.1.2.0. Atlanta: Centers for Disease Control and Prevention; 2017.

13. Secretaria de Estado da Saúde de São Paulo (São Paulo). Plano Estadual de Saúde - PES 2016 - 2019:2015. São Paulo: Secretaria de Estado de Saúde; 2015.
14. Ribeiro MDA, Silva JCA, Oliveira SB. Estudo epidemiológico da hanseníase no Brasil: reflexão sobre as metas de eliminação. Rev Panam Salud Publica 2018; 42(42).

15. Marcondes FL, Tavares CMM, Santos GS, Silva TN, Silveira PG. Capacitação profissional de enfermagem na atenção primária à saúde: Revisão integrativa. Revista Pró-Univer SUS. 2015.; 06(3):09-15.

16. Batista KBS, Gonçalves OSJ. Formação dos profissionais de saúde para o SUS: significado e cuidado. Saúde Soc 2011; 20(4):884-899.

17. Oliveira MPR et al. Formação e Qualificação de Profissionais de Saúde: Fatores Associados à Qualidade da Atenção Primária. Revisa Brasileira de Educação Médica 2016; 40(4):547-559.

18. Costa DB, Garcia SD, Vannuchi MTO, Haddad MCL Impacto do treinamento de equipe no processo de trabalho em saúde: revisão integrativa. Rev Enferm UFPE online 2015; 9(4): 7439-47. doi: 10.5205/reuol.727562744-1-SM.0904201532

19. Peixoto LS, Gonçalves LC, Costa TD, Melo CMT, Cavalcanti ACD, Cortez EA. Educación permanente, continuada y de servicio: desvelando sus conceptos. Enferm Glob. 2013; 12(1):307-322.

20. Fagundes NC, Rangel AGC, Carneiro TM, Castro LMC, Gomes BS. Educação permanente em saúde no contexto do trabalho da enfermeira. Rev Enferm UERJ.2016; 24(1):1-6.

21. Savassi LCM, Modena CM. Hanseníase e a atenção primária: desafios educacionais e assistenciais na perspectiva de médicos residentes. Hansen Int. 2015; 40 (2): 2-16.

22. Silva JAM, Ogata MN, Machado MLT. Capacitação dos trabalhadores de saúde na atenção básica: impactos e perspectivas. Rev Eletrônica Enferm. 2007; 9(2): 389-40.

23. Vieira NF, Lanza FM, Martinez-Riera JR, Nolasco A, Lana FCF. Orientación de la atención primaria em las acciones contra la lepra: factores relacionados con los profesionales. Gac Sanit. 2020; 34(2):120-126.

24. Araújo KMFA, Leano HAM, Rodrigues RN, Bueno IC, Lana FCF. Tendência de indicadores epidemiológicos da hanseníase em um estado endêmico. Rev Rene. 2017; 18(6):771-778 
25. Ministério da Saúde (BR). Secretaria de Vigilância em Saúde. Departamento de Vigilância Epidemiológica. Guia de procedimentos técnicos: baciloscopia em hanseníase. Brasília: Ministério da Saúde, 2010.

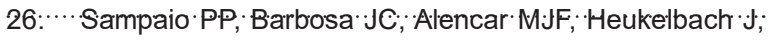
Hinders D, Magalhães MC, Ramos-Jr AN. Programa de Controle da Hanseníase no Brasil: avaliação por profissionais de saúde. Cad Saúde Coletiva. 2009; 17(1): $273-287$.

27:.... Ministério da Saúde (BR): Portaria n n.198 GM/MS de 13 de fevereiro de 2004. Institui a Política Nacional de Educação Permanente em Saúde como estratégia do Sistema Único de Saúde para a formação e o desenvolvimento de trabalhadores para o setor e dá outras providências. Diário Oficial da União, Brasília (DF), 2004 fev 32; Seção, 1:37-41.
28. Ministério da Saúde (BR). Portaria $n^{\circ} 1.996$ GM/MS, de 20 de agosto de 2007. Dispõe sobre as diretrizes para a implementação da Política Nacional de Educação Permanente em Saúde. Diário Oficial da União, Brasília (DF), 2007 ago 162; Seção 1:34-38.

\section{CORRESPONDÊNCIA}

Susilene Maria Tonelli Nardi Instituto Adolfo Lutz, Rua Alberto Sufredini Bertoni, 2325 Vila Maceno,

São José do Rio Preto, São Paulo, Brasil. CEP: 15.060-020.

E-mail: susilene.nardi@ial.sp.gov.br 